\title{
La educación en derechos humanos: un aporte al posconflicto
}

\author{
Andrea Ximena Calderón-Martínez ${ }^{*}$
}

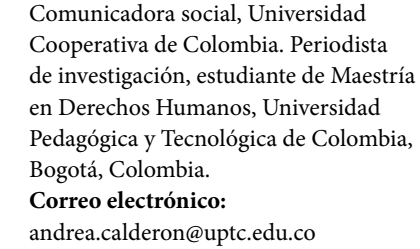

Comunicadora social, Universidad Cooperativa de Colombia. Periodista de investigación, estudiante de Maestría en Derechos Humanos, Universidad Pedagógica y Tecnológica de Colombia, Bogotá, Colombia.

Correo electrónico:

andrea.calderon@uptc.edu.co

Recibido: 30 de agosto del 2016 Aprobado: 26 de septiembre del 2016

Cómo citar este artículo: Andrea Ximena Calderón-Martínez. La educación en derechos humanos: un aporte al posconflicto. DIXI 25. Enero 2017. Págs. 41-48. doi: http:// dx.doi.org/10.16925/di.v19i25.1819di. v19i25.1818

\section{Resumen}

Propósito: actualmente, Colombia atraviesa por un momento histórico enmarcado por un proceso de paz que, de lograrse, propiciará una etapa quizás más compleja que la misma guerra a la que se le pretende dar fin. De manera que se hace preciso destacar el papel fundamental que cumple la educación en derechos humanos, en el propósito de enfrentar ese nuevo rumbo. Temas: en tiempos de posguerra, se hace imprescindible asignarles especial atención a temas como la educación y la pedagogía, ambos dentro de un contexto de derechos humanos que, en tiempos de conflicto, fueron vulnerados a gran escala. Desarrollo: un breve recorrido por la historia de la educación en derechos humanos, sumado a la experiencia de algunos países que ya han atravesado un periodo de posconflicto, ponen de manifiesto que la formación en DD. HH. es clave para mejorar la convivencia y la paz, más aún, en vísperas de un posible periodo de posconflicto en Colombia. Conclusión: se dilucida la relevancia de la implementación de la educación en derechos humanos como un factor determinante, a fin de que se hagan precisos esfuerzos sustanciales que permitan la promoción de una cultura de paz.

Palabras clave: convivencia, cultura de paz, derechos humanos, educación, pedagogía, posconflicto. 


\title{
Human rights education: A contribution to post-conflict
}

\begin{abstract}
Purpose: Colombia is currently going through a historical moment framed by a peace process that, if achieved, will lead to a perhaps more complex stage than the very war it intends to end. Thus, it is necessary to emphasize the fundamental role played by human rights education in facing this new course. Themes: in post-war times, it is essential to pay special attention to issues such as education and pedagogy, both within a context of human rights that, in times of conflict, were violated on a large scale. Development: a brief overview of the history of human rights education, coupled with the experience of some countries that have already gone through a period of post-conflict, show that human rights education is key to improving coexistence and peace, especially on the eve of a potential post-conflict period in Colombia. Conclusion: the relevance of implementing human rights education is elucidated as a determining factor so that substantial efforts are made to promote a peace culture.
\end{abstract}

Keywords: education, coexistence, peace culture, human rights, pedagogy, post-conflict.

\section{A educação em direitos humanos: uma contribuição para o pós-conflito}

\section{Resumo}

Propósito: atualmente, a Colômbia passa por um momento histórico marcado por um processo de paz que, se for atingido, propiciará uma etapa provavelmente mais complexa do que a própria guerra à qual se pretende finalizar. Portanto, fazse necessário destacar o papel fundamental que a educação em direitos humanos desempenha no propósito de enfrentar esse novo rumo. Temas: em tempos de pós-guerra, é imprescindível designar especial atenção a temas como a educação e a pedagogia, ambos no contexto de direitos humanos que, em tempos de conflito, foram violados a grande escala. Desenvolvimento: um breve percorrido pela história da educação em direitos humanos somado à experiência de alguns países que já atravessaram um período de pós-conflito evidenciam que a formação em direitos humanos é fundamental para melhorar a convivência e a paz, mais ainda, perto de um possível período de pós-conflito na Colômbia. Conclusão: elucida-se a relevância da implantação da educação em direitos humanos como um fator determinante a fim de que façam precisos esforços substanciais que permitam a promoção de uma cultura de paz.

Palavras-chave: convivência, cultura de paz, direitos humanos, educação, pedagogia, pós-conflito. 


\section{INTRODUCCIÓN}

A partir del 2012 iniciaron los diálogos de paz entre la administración de Juan Manuel Santos y las Fuerzas Armadas Revolucionarias de Colombia (FARC), con el fin de lograr, según el actual gobierno, la terminación del conflicto con dicho grupo insurgente, en guerra con el Estado durante más de cinco décadas.

Según los registros de la Unidad de Víctimas, el conflicto armado interno ha dejado como saldo, desde 1985, alrededor de ocho millones de víctimas, siendo el desplazamiento forzado, el homicidio, las mutilaciones por minas, el secuestro, la tortura, el reclutamiento de menores, el despojo de tierras, la agresión sexual, las amenazas y los atentados, la desaparición forzada y el robo de bienes, las transgresiones más relevantes. ${ }^{1}$

Un país cuya historia está atravesada por un conflicto que ha dejado una cifra de víctimas tan perturbadora como la anteriormente mencionada, realmente necesita, cuando menos, la esperanza de que exista un escenario en el que impere la paz. Basta con la decisión del pueblo para darle un rumbo diferente al país, que en los últimos gobiernos ha estado girando en torno a las FARC.

Justamente, el tema de los diálogos de paz, cuyo contenido ha sido el centro de un debate tanto ideológico, como político, y el eje de una irrebatible polarización, acéptese o no, es un proceso que se puede marcar como un "hecho histórico para Colombia".

Con relación al posconflicto conviene decir que un factor de gran importancia a la hora de enfrentar un escenario de posguerra es la educación, para ser más exactos, la educación en derechos humanos.

\section{CONTEXTO HistóRICO Y JURÍDICO EN TORNO A LA CONSTRUCCIÓN DE LA EDUCACIÓN EN DERECHOS HUMANOS}

Desde la Declaración Universal de los Derechos Humanos, proclamada por la Asamblea General de las Naciones Unidas el 10 de diciembre de 1948, los derechos humanos y la educación se encuentran estrechamente unidos. En este documento no solo se

\footnotetext{
1. Véase Eltiempo.com. Víctimas del conflicto en Colombia ya son ocho millones. Desplazamiento, amenazas y despojo, los hechos violentos más denunciados. Eltiempo.com. Abril 16 del 2016. Disponible en http://www.eltiempo.com/politica/justicia/cifras-de-las-victimasdel-conflicto-armado-en-colombia/16565045

2. Véase Telemedellin.tv. Proceso de paz es un logro histórico para Colombia: Fiscal de la CPI. Telemedellin.Tv. Disponible en http:// telemedellin.tv/proceso-de-paz-fiscal-de-la-cpi/
}

establece la educación como un derecho universal, así también los hace el artículo 26:

La educación tendrá por objeto el pleno desarrollo de la personalidad humana y el fortalecimiento del respeto a los derechos humanos y a las libertades fundamentales; favorecerá la comprensión, la tolerancia y la amistad entre todas las naciones y todos los grupos étnicos o religiosos, y promoverá el desarrollo de las actividades de las Naciones Unidas para el mantenimiento de la paz. ${ }^{3}$

Es a partir de allí que se recomienda a todos los Estados que enseñen, propaguen y expliquen la declaración. Ya en 1950 se invitó a la Organización de las Naciones Unidas para la Educación, la Ciencia y la Cultura (Unesco), a fomentar y facilitar la enseñanza de los derechos humanos en las escuelas, los programas de educación para adultos y los medios de comunicación. Lo anterior con el fin de promover la educación en derechos humanos, como factor indispensable para el mejoramiento de la calidad del proceso formativo y consolidar una cultura democrática, pacífica y respetuosa.

Además de la Declaración Universal, el artículo 13.1 del Pacto Internacional de Derechos Económicos, Sociales y Culturales celebrado en 1966, reconoce el derecho de toda persona a la educación y a la educación en derechos humanos, por catalogarla como un medio adecuado para promover la participación de cada uno dentro de la sociedad. El artículo establece lo siguiente:

Los Estados-partes del presente Pacto reconocen el derecho de toda persona a la educación. Concuerdan en que la educación deberá tender al desarrollo pleno de la personalidad humana y de su dignidad y fortalecer el respeto por los derechos humanos y por las libertades fundamentales. Concuerdan, también, en que la educación deberá capacitar a todas las personas para que participen de manera efectiva de una sociedad libre, además de favorecer la comprensión, la tolerancia, la amistad entre todas las naciones y entre todos los grupos radicales, étnicos o religiosos, y promover las actividades de las Naciones Unidas en pro del mantenimiento de la paz. ${ }^{4}$

\footnotetext{
3. Véase Un.org. Declaración Universal de Derechos Humanos. UN.ORG. Disponible en http://www.un.org/es/documents/udhr/ index_print.shtml

4. Véase Ohchr.org. Pacto Internacional de Derechos EcoNómicos, Sociales y Culturales. Adoptado y abierto a la firma, ratificación y adhesión por la Asamblea General en su resolución $2200 \mathrm{~A}$ (xxI), de 16 de diciembre de 1966. Entrada en vigor: 3 de
} enero de 1976, de conformidad con el artículo 27. OHCHR.ORG. 
A fin de fomentar la educación en derechos humanos, la Unesco aprobó, en 1974, la "Recomendación sobre la educación para la comprensión, la cooperación y la paz internacionales y la educación relativa a los derechos humanos y los derechos fundamentales". La recomendación destaca la importancia ${ }^{5}$ "del conocimiento, no solamente de los derechos, sino también de los deberes que tienen las personas, los grupos sociales y las naciones, para con los otros" (Párrafo 4).

Otro documento de gran importancia es el que se ejecutó en la Conferencia Mundial de los Derechos Humanos, llevado a cabo en Viena en 1993. Con respecto a la educación, específicamente el artículo 33, reafirma el deber de los Estados de "orientar la educación para el fortalecimiento del respeto a los derechos humanos y las libertades fundamentales". La declaración resalta también la importancia de "incorporar el tema de los derechos humanos en los programas educacionales", y solicita a los Estados que procedan en dicho sentido, subrayando que: "La educación en derechos humanos y la divulgación de informaciones adecuadas, tanto de carácter teórico como de carácter práctico, desempeñan un papel importante en la promoción y el respeto hacia los derechos humanos de todos los individuos, sin distinción". ${ }^{6}$ Este plan de acción, a su vez, dedica un apartado a la educación en derechos humanos, comprendidos desde el punto 78 al 82, en los que se resalta la responsabilidad que tienen los Estados. En el punto 79 se solicita "la inclusión de los derechos humanos, el derecho humanitario, la democracia y el imperio de la ley como temas de los programas de estudio de todas las instituciones de enseñanza académica y no académica"?

Por su parte, la observación general N. ${ }^{\circ} 13$ del Pacto Internacional de Derechos Económicos Sociales y Culturales de 1999, en el artículo 13 hace referencia a la importancia del derecho a la educación, y destaca: "La educación es un derecho humano intrínseco y un medio indispensable para hacer efectivos otros derechos humanos".

Disponible en http://www.ohchr.org/SP/ProfessionalInterest/Pages/ CESCR.aspx

5. Véase Susana Beatriz Sacavino. Democracia y educación EN Derechos humanos en América Latina. Ediciones Desde Abajo. (2012). Pág. 65.

6. Id. Págs. 63-64.

7. Véase Ohchr.org. Declaración y Programa de Acción DE VIENA. OHCHR.ORG. Disponible en http://www.ohchr.org/Documents/ Events/OHCHR20/VDPA_booklet_Spanish.pdf.Pág. 50

8. Véase Escr-net.org. Observación general $N^{\circ}$ 13: El derecho a la educación (artículo 13). EsCR-NRGET.O. Disponible en https:// www.escr-net.org/es/recursos/observacion-general-no-13-derecho-educacion-articulo-13
Entre tanto, el Alto Comisionado de las Naciones Unidas para los Derechos Humanos llevó a cabo el Plan de Acción del Decenio, del que se desprende el Programa Mundial para la Educación en Derechos Humanos (primera etapa, 2005-2007), el cual fue proclamado por la Asamblea General de la onu el 10 de diciembre del 2004. Los objetivos de dicho plan son: a. Contribuir a forjar una cultura de derechos humanos; b. Promover el entendimiento común, sobre la base de los instrumentos internacionales, de los principios y metodologías básicos para la educación en derechos humanos; c. Asegurar que la educación en derechos humanos reciba la debida atención en los planos nacional, regional e internacional; $d$. Proporcionar un marco colectivo común para la adopción de medidas a cargo de todos los agentes pertinentes; e. Ampliar las oportunidades de cooperación y asociación en todos los niveles; y f. Aprovechar y apoyar los programas de educación en derechos humanos existentes, poner de relieve las prácticas satisfactorias y dar incentivos para continuarlas o ampliarlas y crear prácticas nuevas. ${ }^{9}$

Las herramientas anteriormente mencionadas, todas pertenecientes a la esfera internacional, llevaron a que en Colombia se concretara lo que hoy se conoce como el Plan Nacional de Educación en Derechos Humanos (Planedh), el cual apunta a "la transformación social, al empoderamiento de la sociedad para la realización de sus derechos y libertades y al fortalecimiento de las capacidades de hombres y mujeres para afrontar la defensa y ejercicio de los mismos" ${ }^{10}$

\section{LA EDUCACIÓN EN DD. HH. COMO} EFECTO DE LA GUERRA

Lamentablemente, la aparición de la formación en DD. HH. en Latinoamérica es producto, justamente, de las violaciones de los derechos fundamentales, especialmente en épocas de dictadura como ocurrió en Chile, Argentina y Perú, entre otros. Fue entonces, a finales de la década de los ochenta, cuando surgió en América Latina la educación en/para los derechos humanos, expresión que Sacavino consideró pertinente, ${ }^{11}$ ya que

\footnotetext{
9. Véase Ohchr.org. Plan de Acción. Programa Mundial Para la Educación en Derechos Humanos. Primera etapa. OHCHr.org. Disponible en http://www.ohchr.org/Documents/ Publications/PActionEduc ationsp.pdf Pág. 15.

10. Véase Ohchr.org. Plan Nacional de Educación en DereCHOS Humanos. OHCHR.org. Disponible en http://www.ohchr.org/ Documents/Issues/Education/Training/actions-plans/Colombia. pdf

11. Véase Susana Beatriz Sacavino, supra, nota 5. Pág 23.
} 
el acto de educar lleva consigo un aspecto importante que es el de saber, el de informarse, el de comprender el contenido de los derechos humanos y su significado jurídico, histórico y social, entre otros. ${ }^{12}$

Hay que tener presente que la ola de violencia no solo azota en tiempos de conflicto, ya que en un escenario de posguerra es probable que la coacción llegue a aumentar, como ocurrió con Guatemala y El Salvador. El conflicto bélico interno ocurrido en el territorio salvadoreño tuvo lugar desde 1979 hasta 1992, y el conflicto armado interno en Guatemala duró 36 años.

Los procesos de paz de El Salvador y Guatemala fueron la salida a prolongados e intensos conflictos armados que azotaron la región centroamericana. Los distintos actores consideraron este momento como el punto de partida para el establecimiento de un proyecto estatal que superara las causas del conflicto, y llevara a la población a una convivencia sana en el posconflicto. Sin embargo, este escenario de posconflicto, lejos de convertirse en un periodo de paz, llevó a estos países a unos niveles de violencia que llegaron a ubicarlos entre los más altos del mundo. La consecución de los procesos de paz y el fin del conflicto armado no significaron para los Estados centroamericanos la terminación de los enfrentamientos sociales. ${ }^{13}$

Luego del proceso de paz que se logró ejecutar en Guatemala, la violencia persistió y aumentaron de forma alarmante delitos como el secuestro, el robo de vehículos y residencias y los asaltos en los buses, entre otros. Así, "en este escenario, se hace evidente que la violencia como fenómeno social ligado a la delincuencia común continúa presente y solamente se modificaron los actores y sus intereses". ${ }^{14}$ Mientras tanto, en El Salvador, según documentó la Fiscalía General de la República:

Los homicidios ascendieron de 8.019 en 1996 a 8.281 en 1998 (cabe resaltar que, durante los doce años de guerra civil, perdieron la vida en promedio anual 6.330 personas). En 1998, seis años después de haber sido firmado el acuerdo de paz, este país se ubicó como el más violento de América Latina. ${ }^{15}$

\footnotetext{
12. Id.

13. Véase Camilo Andrés Devia Garzón, Dina Alejandra Ortega Avellaneda y Marcela Julieth Magallanes Montoya. Violencia luego de la paz: escenarios de posconflicto en Centroamérica. Revista Republicana. 2014. Pág. 130.

14. Véase ojs.urepublicana.edu.co/index.php/revistarepublicana/ article/download/14/13

15. Id. Pág. 131
}

Pese a que la paz es un estado al que aspira un país como Colombia, salpicado por la guerra durante más de 50 años, hay que tener presente que el periodo de posconflicto no es tan seguro como se cree. Sin embargo, el panorama se hace alentador en la medida en que el Estado, las diferentes organizaciones y todos los actores implicados directa o indirectamente, tomen medidas que estén a la altura de un proceso de paz que algunas destacadas personalidades han catalogado como "ejemplo para el mundo". ${ }^{16}$

Cabe recalcar que no todos los procesos de posguerra están relacionados estrictamente con el tema de la violencia. Se puede poner sobre la mesa el caso de Irlanda del Norte, cuyo conflicto armado - netamente interétnico- duró casi tres décadas. Este es un claro ejemplo de la importancia de la educación en derechos humanos y/o paz, tras el fin de la guerra. El país norirlandés creó el Foro para la Educación en la Comprensión Mutua y en la Herencia Cultural (EMU/CH, en inglés, Education for Mutual Understanding \& Cultural Heritage). En este foro, además de la producción de material pedagógico, se organizan actividades por medio de diferentes expresiones artísticas como la música, la danza, los cuentos y las leyendas, que denotan un pasado cultural en común, y los niños, sin importar si son católicos o protestantes, se unen..$^{17}$ Desde un principio, la aspiración de este proceso se enfocó en desafiar identidades y creencias sectarias que no permitían ningún tipo de integración con el otro, a quien, de facto, se percibía como un enemigo. En concreto, se buscaba suscitar la integración, expresamente el reconocimiento.

Así, se empezó a acercar, pues, la situación que actualmente atraviesa Colombia y el desafío que se avecina:

El reto estaría en la capacidad de reconocer las dimensiones que ha tenido el conflicto tanto en el campo como en las ciudades, así como en el dolor de las víctimas en su amplio universo, de modo que no se mire a estas solidariamente según el victimario. ${ }^{18}$

\footnotetext{
16. Véase Diariolasamericas.com. Proceso de paz de Colombia es un "ejemplo para el mundo", dice Humala. Diariolasamericas.com. Disponible en http://www.diariolasamericas.com/america-latina/ proceso-paz-colombia-es-un-ejemplo-el-mundo-dice-humala-n3431372

17. Véase Rosa Carbonell de la Puente. Educar para La PAZ EN IrLANDA DEL Norte. LA AUtORA COMPARTE LA EXPERIENCIA DE HABER PARTICIPADO EN UN SEMINARIO SOBRE LA EDUCACIÓN PARA la Paz, en Belfast en abril de 1998. Escuela Abierta, 2. (1998). Pág. 170.

18. Véase también Elespectador.com. El papel de la educación en el posconflicto. La educación es un factor determinante tanto en la guerra y como en la paz. Elespectador.com. Agosto 26 de 2015. Disponible en http://www.elespectador.com/opinion/
} 
Tras haber mencionado con anterioridad la relevancia que a lo largo de la historia ha venido adquiriendo la formación en derechos humanos, se llega de nuevo a los acuerdos de paz. Entonces, es fácil comprender por qué, en lo pactado en La Habana, uno de los temas cruciales fue el de la educación en DD. HH. Respecto al tema, el acuerdo final entre Gobierno y las FARC hace referencia en uno de los ítems del punto 5.2.1; esto es, al fortalecimiento de los mecanismos de promoción de los derechos humanos y la consolidación del proceso de implementación del Plan Nacional de Educación en Derechos Humanos, entre otros:

- La inclusión del Acuerdo Final y del Informe Final de la Comisión para el Esclarecimiento de la Verdad, la Convivencia y la No Repetición al Plan Nacional de Educación en Derechos Humanos.

- La articulación de los programas de promoción de cultura política democrática y participativa del Punto 2 "Participación Política: Apertura democrática para construir la paz", en particular para la superación de la estigmatización asociada al conflicto.

- El fortalecimiento de las medidas de educación no formal a través de la puesta en marcha de campañas públicas de reconocimiento de los derechos humanos y prevención de su violación. ${ }^{19}$

\section{CONCLUSIONES}

Se puede aseverar, entonces, que la afirmación de los derechos humanos como tema de interés internacional se fundamenta, estratégicamente, en la percepción según la cual las fuertes violaciones pueden llevar a la guerra. ${ }^{20}$ De manera que, por simplicidad, es posible suponer que, tras la salida negociada del conflicto en Colombia, se hace imprescindible una verdadera transformación del Estado y también de la sociedad. Por ello, debo destacar el papel fundamental que cumple el campo pedagógico como herramienta dinamizadora y creadora de nuevos espacios de participación en una nueva realidad de posconflicto.

Sin duda, un reto para la construcción de paz es el de desarrollar estrategias pedagógicas para enseñar sobre la historia de la violencia y los conflictos colombianos, así como producir empatía y un

\footnotetext{
el-papel-de-educacion-el-posconflicto

19. Véase ACUERDo FINAL PARA LA TERMINACIÓN DEL CONFLICTO Y LA CONSTRUCCIÓN DE UNA PAZ ESTABLE Y DURADERA. 24 de agosto de 2016. Pág. 169. Disponible en https://www.mesadeconversaciones.com.co/sites/default/files/24_08_2016acuerdofinalfinalfinal-1472094587.pdf

20. Véase Susana Beatriz Sacavino, supra, nota 5. Pág. 35.
}

pensamiento crítico que los deslegitime y promueva relaciones pacíficas y de reconciliación. Varios grupos, entre ellos el Centro de Memoria Histórica, ya están asumiendo este reto: ${ }^{21}$

Es ahora el momento de convocar y comprometer a todos los agentes personales e institucionales del Sistema Educativo Nacional, para que sin dilaciones injustificables asuman, de verdad, la responsabilidad ya confiada por la Constitución Nacional y las leyes del sector, cual es la de desarrollar y poner en práctica una pedagogía social para la fundamentación y el desarrollo de la dignidad humana en Colombia, y el conocimiento, la valoración y la introyección de los Derechos Humanos en las actitudes y el comportamiento de todos los ciudadanos, empezando por los niños y los jóvenes, haciendo que sean ellos la esperanza cierta de un nuevo país. $^{22}$

La educación en derechos humanos es la vía más racional y prometedora para aprender y tomar conciencia acerca de los valores y principios que enaltecen la dignidad del ser humano, pues estos se han venido transformando en una fuente de inspiración para construir respuestas adecuadas a la creciente complejidad que traen consigo los continuos cambios sociales.

Por ello, se hace imprescindible en el plano educativo en derechos humanos, integrar un panorama diferencial en relación con los derechos esenciales que corresponden a las mujeres, los pueblos indígenas, las comunidades afrocolombianas, el pueblo Rom, las personas en situación de discapacidad, de indigencia o de desplazamiento forzado, a las comunidades LGTBI, la población afectada por la violencia, en condiciones de pobreza extrema, y ahora posiblemente los excombatientes, lo que otorga nuevos significados a los derechos que les son propios, tanto en el ámbito de lo privado, como en el del dominio público.

Cabe aclarar que la educación en derechos humanos no abarca solo la educación formal, ya que existen también otros organismos que desarrollan diferentes programas organizados que van desde la educación informal (como la labor con medios de comunicación y la población en general), hasta la educación para el trabajo y el desarrollo humano, dirigida a servidores

\footnotetext{
21. Véase Uniandes.edu.co. Educación para la paz en tiempos de posconflicto. Uniandes.edu.co. Disponible en https://sextante. uniandes.edu.co/index.php/ejemplares/sextante-6/horizontes/ educacion-para-la-paz-en-tiempos-de-posconflicto

22. Véase Elcolombiano.com. Educación y Posconflicto. Elcolombiano.com. Marzo 3 de 2015. Disponible en http://www. elcolombiano.com/opinion/columnistas/educacion-y-posconflicto -FA1406160
} 
públicos y organizaciones comunitarias y sociales, entre otros. ${ }^{23}$

Una vez Colombia se enfrente al periodo de posguerra, debe estar preparada y dispuesta a dar un paso importante hacia la convivencia pacífica. Con esto en mente, la autora de este artículo se permite citar a Nelson Mandela, quien alguna vez sabiamente precisó: "La educación es el arma más poderosa que puedes usar para cambiar el mundo". Es claro entonces que "el conocimiento de los derechos y las libertades está considerado como un instrumento fundamental para asegurar el respeto de los derechos de todas las personas". ${ }^{24}$

De manera que la responsabilidad para instaurar una cultura de la paz corresponde entonces a la pedagogía, lo que le abre espacio a la formación en derechos humanos, los cuales nacen como fruto del pensamiento libre, por la creencia del respeto de estos y al permitir así aceptar y reconocer las diferencias existentes entre unos individuos y otros.

\section{REFERENCIAS}

ACUERDo FinAl PARA LA TERMINACIÓN DEL CONFLicto Y LA CONSTRUCCIÓN DE UNA PAZ ESTABLE Y DURADERA. 24 de agosto de 2016. Pág. 169. Disponible en https:// www.mesadeconversaciones.com.co/sites/default/files/24_08_2016acuerdofinalfinalfinal-1472094587.pdf

Camilo Andrés Devia Garzón, Dina Alejandra Ortega Avellaneda y Marcela Julieth Magallanes Montoya. Violencia luego de la paz: escenarios de posconflicto en Centroamérica. Revista Republicana. 2014.

Diariolasamericas.com. ProcesodepazdeColombiaesun "ejemplo para el mundo", dice Humala. Diariolasamericas. сом. Disponible en http://www.diariolasamericas.com/ america-latina/proceso-paz-colombia-es-un-ejemplo-el-mundo-dice-humala-n3431372

Elcolombiano.com. Educación y Posconflicto. ElCOLOMBIANO.COM. Marzo 3 del 2015. Disponible en http:// www.elcolombiano.com/opinion/columnistas/educacion-y-posconflicto-FA1406160

23. Véase Colombiaaprende.edu.co. La Educación en Derechos Humanos. ColombiaAprende.edu.co. Disponible en http://www. colombiaaprende.edu.co/html/productos/1685/w3-article-312142 html

24. Véase Unesco.org. Educación para los Derechos Humanos. Unesco. ORG. Disponible en http://www.unesco.org/new/es/education/themes/ leading-the-international-agenda/human-rights-education/
Elespectador.com. El papel de la educación en el posconflicto. La educación es un factor determinante tanto en la guerra y como en la paz. ElespeCtador.com. Agosto 26 de 2015. Disponible en http://www.elespectador.com/ opinion/el-papel-de-educacion-el-posconflictos

Eltiempo.com. Víctimas del conflicto en Colombia ya son ocho millones. Desplazamiento, amenazas y despojo, los hechos violentos más denunciados. ELtiempo.com. Abril 16 del 2016. Disponible en http://www.eltiempo.com/ politica/justicia/cifras-de-las-victimas-del-conflicto-armado-en-colombia/16565045

Escr-net.org. Observación general No 13: El derecho a la educación (artículo 13). EsCR-NRGET.O. Disponible en https://www.escr-net.org/es/recursos/observacion-general-no-13-derecho-educacion-articulo-13

Ohchr.org. Declaración y Programa de Acción de VieNA. OHCHR.ORG. Disponible en http://www.ohchr.org/ Documents/Events/OHCHR20/VDPA_booklet_Spanish.pdf.

Ohchr.org. Pacto Internacional de Derechos Económicos, Sociales y Culturales. Adoptado y abierto a la firma, ratificación y adhesión por la Asamblea General en su resolución 2200 A (xxI), de 16 de diciembre de 1966. Entrada en vigor: 3 de enero de 1976, de conformidad con el artículo 27. OHCHR.ORG.

Ohchr.org. Plan de Acción. Programa Mundial para la Educación en Derechos Humanos. Primera etapa. OHCHR.ORG. Disponible en http://www.ohchr.org/Documents/ Publications/PActionEduc ationsp.pdf

Ohchr.org. Plan Nacional de Educación en Derechos Humanos. OHChr.org. Disponible en http://www. ohchr.org/Documents/Issues/Education/Training/actions-plans/Colombia.pdf

Rosa Carbonell de la Puente. EduCAR PARA LA PAZ EN IrLANDA DEL NoRTE. LA AUTORA COMPARTE LA EXPERIENCIA DE HABER PARTICIPADO EN UN SEMINARIO SOBRE la Educación para la Paz, en Belfast en abril de 1998. Escuela Abierta, 2. (1998).

Susana Beatriz Sacavino. Democracia y educación en DERECHOS humanos en AmÉrica Latina. Ediciones Desde Abajo. (2012). Pág. 65.

Telemedellin.tv. Proceso de paz es un logro histórico para Colombia: Fiscal de la CPI. TeLEMEdeLLIN.Tv. Disponible en http://telemedellin.tv/proceso-de-paz-fiscal-de-la-cpi/

Un.org. Declaración Universal de Derechos Humanos. UN.ORG. Disponible en http://www.un.org/es/documents/udhr/ index_print.shtml

Unesco.org. Educación para los Derechos Humanos. UnEsCo.org. Disponible en http://www.unesco.org/new/ 
es/education/themes/leading-the-international-agenda/human-rights-education/

Uniandes.edu.co. Educación para la paz en tiempos de posconflicto. Uniandes.eDu.co. Disponible en https:// sextante.uniandes.edu.co/index.php/ejemplares/sextante-6/horizontes/educacion-para-la-paz-en-tiempos-de-posconflicto 